\title{
Eclampsia with Posterior Reversible Encephalopathy Syndrome
}

\author{
Kyawzaw Lin ${ }^{1}$, Aung Naing Lin ${ }^{1}$, Sithu Lin ${ }^{1}$, Thinzar Lin ${ }^{1}$, Khin Myint ${ }^{1}$, Jose Cabassa ${ }^{2}$ \\ Departments of ${ }^{1}$ Internal Medicine and ${ }^{2}$ Neurology, The Brooklyn Hospital Center, Affiliate of the Mount Sinai Hospital, 121, \\ Dekalb Avenue, Brooklyn, NY 11201.
}

\section{Corresponding Author:}

Dr. Kyawzaw Lin

Email: dr.kyawzawlin2015@gmail.com

This is an Open Access article distributed under the terms of the Creative Commons Attribution License (creativecommons.org/ licenses/by/3.0).

Received Accepted Published

\begin{abstract}
Background: The posterior reversible encephalopathy syndrome (PRES) or reversible posterior leukoencephalopathy syndrome (RPLS) includes headache, visual disturbance, and seizure, altered mental status with or without focal neurological signs. Here, we present a 24 year old postpartum female with PRES in the setting of post-partum eclampsia with cortical blindness and seizure. Case Report: A 24 year old African American female presented with severe, constant, throbbing, bilateral occipital headache nine days after normal spontaneous vaginal delivery followed by blurred vision for two days. It was complicated by generalized tonic-clonic witnessed seizure. Magnetic Resonance Imaging (brain) showed findings consistent with posterior reversible encephalopathy syndrome. The patient was successfully managed with intravenous labetolol and magnesium sulfate for eclampsia in intensive care unit. Conclusion: Focussed clinical approach, early detection of significant neuroimaging findings upon presentation, timely aggressive treatment and cessation of causal agents are crucial for satisfactory recovery and outcomes. This syndrome is overlooked by the physicians and usually radiologists are the saviors for timely diagnosis for PRES.
\end{abstract}

Keywords: Eclampsia, Magnetic Resonance Imaging, Posterior Leukoencephalopathy Syndrome, Pregnancy, Seizures.

\section{Introduction}

The posterior reversible encephalopathy syndrome (PRES) is a clinical syndrome of confusion, seizure, headache, visual abnormalities such as hemianopsia, cortical blindness in the setting of pre-eclampsia, eclampsia, infection, autoimmune disease, vasculitis, hypo-magnesemia, hypercalcemia, dialysis, immune suppression or chemotherapy with cyclosporine, cisplatin, tacrolimus or post-transplantation [1]. The neuroimaging findings are reversible subcortical edema without obvious infarction.

\section{Case Report}

24 year old African American female presented with severe, constant, throbbing, bilateral occipital headache nine days after normal spontaneous vaginal delivery followed by blurred vision for two days. The patient was admitted to Obstetrics unit for pre-eclampsia and blood pressure was uncontrolled during last week of pre-partum and post-partum period. It was complicated by generalized tonic-clonic witnessed seizure that lasted less than one minute with persistent visual loss and/or disturbance. Although she had difficulty in describing the visual problems, she was able to see the objects but could not make up what they were. She denied chest pain, palpitation, shortness of breath, dizziness, nausea, vomiting, abdominal pain, tingling and numbness sensation, dysuria or localized weakness or unstable gait.

Complete blood count, basic metabolic panel, liver function and coagulation profile were within normal limit. Electrocardiogram showed 
sinus bradycardia at $50 \mathrm{bpm}$. Trans-esophageal echocardiogram showed normal ejection fraction and no atrial septum defect or ventricular septum defect. Computed Tomography scan (head) without contrast [Fig.1] showed normal and repeated CT scan [Fig.2] showed no evidence of acute intracranial hemorrhage, fluid collections or mass effect. Magnetic Resonance Imaging (brain) [Fig.3,4] showed cortical and sub-cortical regions with abnormal FLAIR (Fluid Attenuated Inversion Recovery) signal with associated bright diffusion signal involving the frontal and parietal lobes as well as the occipital lobes and left cerebellar hemisphere consistent with posterior reversible encephalopathy syndrome (PRES). The eclampsia was managed with intravenous labetolol and magnesium sulfate with prophylactic levetiracetam. Next morning, her vision improved but still had difficulty seeing the features of a person's face. She was discharged home with instructions to follow up with neurologist.

\section{Discussion}

The posterior reversible encephalopathy syndrome (PRES) or reversible posteriorleukoencephalopathy syndrome (RPLS) is usually neuro-imaging finding of reversible vasogenic subcortical tissue swelling without gross infarction. The underlying patho-physiological hypothesis is (i) cerebral ischemia from vasospasm (ii) disordered cerebral autoregulation and (iii) endothelial dysfunction $[1,2]$. The incidence is commonin young than elderly and in female than male. It is usually presents with clinical seizure in $87 \%$, headache unresponsive to medications in 53\%, encephalopathy in $92 \%$ and visual disturbances in 39\% [3].

\section{Typically, Computed Tomography} scan is less significant in PRES but Magnetic Resonance Imaging (MRI) of the brain demonstrates symmetric, posterior occipito-parieto-temporal area white matter edema. However, they may present with atypical locations or asymmetric lesions. The involvement of frontal lesions is common although

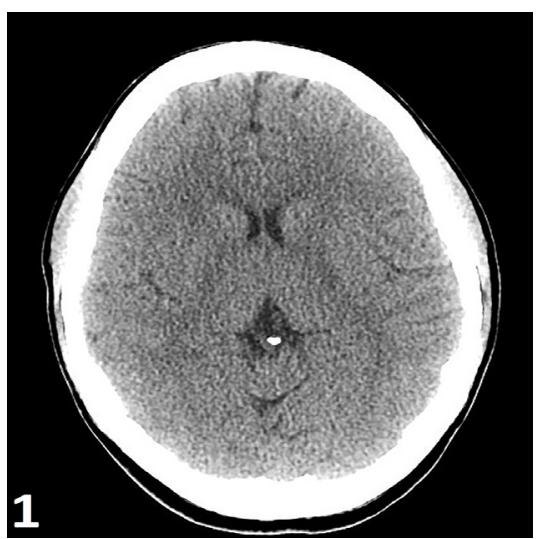

Fig.1: CT scan without contrast (on admission) showed normal the ventricles, sulci and cisterns without evidence of hydrocephalus.

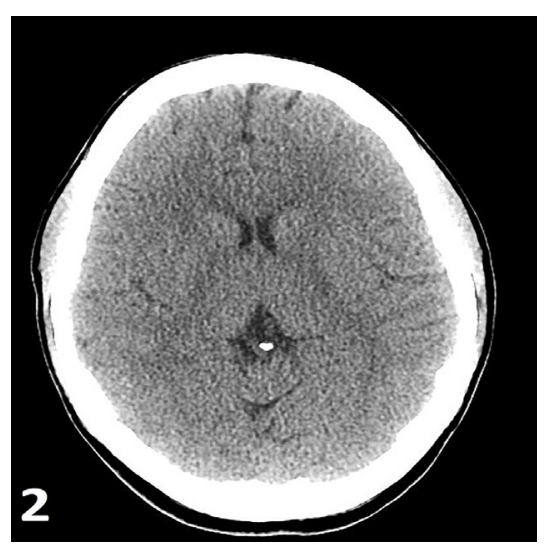

Fig.2: CT scan without contrast (repeated) showed no suspicious interval change.

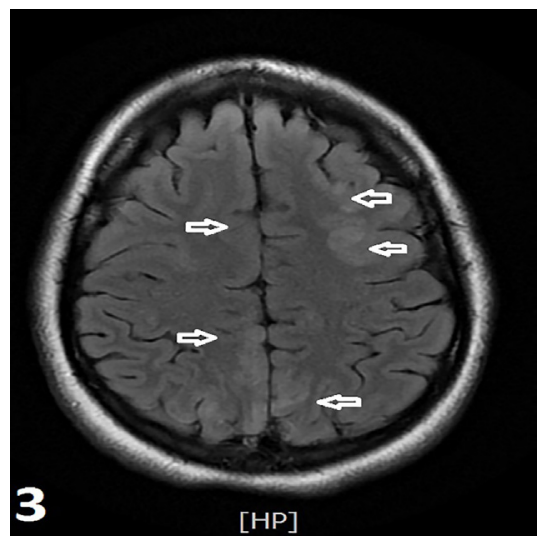

Fig.3: MRI without contrast showed cortical and sub-cortical regions with abnormal FLAIR signal with associated bright diffusion signal (white arrows) involving the frontal, parietal lobes, the occipital lobes and left cerebellar hemisphere consistent with posterior reversible encephalopathy syndrome. 
the lesions may be detected in the brainstem and basal ganglia [4]. MRI showed increased T2 and FLAIR (Fluid Attenuated Inversion Recovery) signal due to cerebral edema in the posterior region of the cerebral hemisphere especially the posterior parieto-occipital lobes as those areas are less sympathetic innervated. The increased signal is usually bilateral and symmetrical but sometimes it may be single and unilateral [5]. In approximately $56 \%$ of the patients, the regions of vertebra-basilar territory such as thalamus and cerebellum are involved [6]. Increased T2 and DWI (diffusion weighted imaging) signal seen as water content in vasogenic edema is high. In setting of vasogenic edema in PRL, DWI is decreased while mobility is increased but in cytotoxic cerebral edema, DWI is increased but water mobility is decreased owing to cerebral edema.

Whenever PRES is in doubt, management should be aggressive as delayed treatment might end up with permanent brain damage. Treatment includes proper blood pressure control, cessation of causal agent or therapeutic agent; manage underlying co-morbidities and seizure prophylaxis. In patient with hypertensive encephalopathy, mean arterial pressure must reduce $20-25 \%$ in first two hours and diastolic pressure to $100 \mathrm{mmHg}$ as rapid reduction in blood pressure worsens end organ perfusion and cerebral function.

\section{Conclusion}

In our case of PRES due to eclampsia, magnesium sulfate is preferred agent for prophylactic seizure owing to neuro-protective effects by stabilizing of cerebral circulation and stabilization of neuronal membranes and inhibiting in stimulatory neuron transmitters, such as glutamate [7,8] and magnesium is superior to diazepam and phenytoin. For PRES due to pre-eclampsia, the mainstay is delivery of placenta and fetus. Most PRES patients will have complete neurological recovery usually in two weeks as in our patient and seizure is in remission once neuroimaging changes have

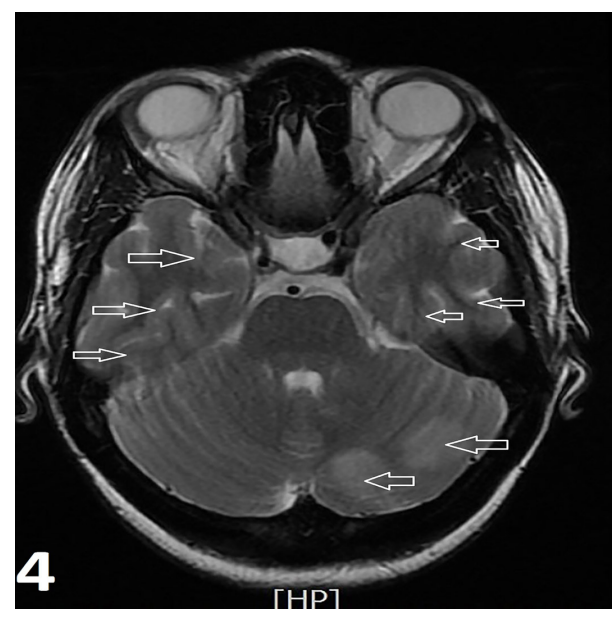

Fig.4: MRI without contrast showed cortical and sub-cortical regions with abnormal FLAIR signal with associated bright diffusion signal (white arrows) involving the frontal, parietal lobes, the occipital lobes and left cerebellar hemisphere consistent with posterior reversible encephalopathy syndrome.

recovered in three to seven days [9]. Complete reversibility is pathognomonic features of PRES or RPLS. However, some cases can progress to leukomalacia and permanent cerebral ischemia and damage particularly in setting of PRES due to immunotherapeutic agents [10].

Contributors: KL contributed in gathering information, writing introduction, case report and discussion. ANL, SL, TL, KM: manuscript editing; JC provided patient care and the discussion of the case. KL will act as guarantor. All authors approved the final version of this manuscript.

Funding: None; Competing interests: None stated.

\section{References}

1. Fugate JE, Claassen DO, Cloft HJ, Kallmes DF, Kozak OS, Rabinstein AA. Posterior reversible encephalopathy syndrome: associated clinical and radiologic findings. Mayo Clinic Proceedings. 2010;85:427-432.

2. Hinchey J, Chaves C, Appignani B, Breen J, Pao L, Wang $\mathrm{A}$, et al. A reversible posterior leukoencephalopathy syndrome. New England Journal of Medicine. 1996;334:494-500.

3. Lee VH, Wijdicks EF, Manno EM, Rabinstein AA. Clinical spectrum of reversible posterior leukoencephalopathy syndrome. Archives of Neurology. 2008;65:205-210.

4. Bartynski WS. Posterior reversible encephalopathy syndrome, part 1: fundamental imaging and clinical 
features. American Journal of Neuroradiology. 2008;29:1036-1042.

5. Mukherjee P, McKinstry RC. Reversible posterior leukoencephalopathy syndrome: evaluation with diffusion-tensor MR imaging. Radiology. 2001;219:756765.

6. Casey SO, Sampaio RC, Michel E, Truwit CL. Posterior reversible encephalopathy syndrome: utility of fluidattenuated inversion recovery $\mathrm{MR}$ imaging in the detection of cortical and subcortical lesions. American Journal of Neuroradiology. 2000;21:1199-1206.

7. Mirzamoradi M, Hosseini MS, Saleh M, Esmaeili S. Posterior reversible encephalopathy syndrome (PRES) associated with eclampsia: A case study. International Journal of Medical Research \& Health Sciences. 2017;6:48-53.

8. Sibai BM. Treatment of hypertension in pregnant women. New England Journal of Medicine. 1996;335:257-265.

9. Garg RK. Posterior leukoencephalopathy syndrome. Postgraduate Medical Journal. 2001;77(903):24-28.

10. Schwartz RB, Bravo SM, Klufas RA, Hsu LI, Barnes $\mathrm{PD}$, Robson CD, et al. Cyclosporine neurotoxicity and its relationship to hypertensive encephalopathy: CT and MR findings in 16 cases. AJR. American journal of Roentgenology. 1995;165:627-631. 